\title{
A Summary on Polycystic Ovary Syndrome: Diagnostic Criteria, Prevalence, Clinical Manifestations, and Management According to the Latest International Guidelines
}

Adriana Catharina Helena Neven, BSC, MSC ${ }^{1}$ Joop Laven, MD, $\mathrm{PhD}^{1}$ Helena J. Teede, MBBS, FRACP, PhD $^{2}$ Jacqueline A. Boyle, MBBS, FRANZCOG, MPH\&TM, PhD 2,3

${ }^{1}$ Department of Obstetrics and Gynecology, Erasmus University Medical Center, Rotterdam, The Netherlands

2 Monash Centre for Health Research and Implementation, Monash Public Health and Preventive Medicine, Monash University, Clayton, Victoria, Australia

${ }^{3}$ Monash Department of Obstetrics and Gynaecology, Monash

Health, Clayton, Victoria, Australia

Semin Reprod Med 2018;36:5-12
Address for correspondence Jacqueline A. Boyle, MBBS, FRANZCOG, MPH\&TM, PhD, Locked Bag 29, Clayton, Victoria 3168, Australia (e-mail: jacqueline.boyle@monash.edu).

\begin{abstract}
Keywords

- PCOS update

- PCOS diagnosis

- PCOS treatment
\end{abstract}

Affecting 8 to $13 \%$ of women of reproductive age and $21 \%$ in high risk groups, ${ }^{1}$ polycystic ovary syndrome (PCOS) is the most prevalent reproductive disorder causing significant health consequences for women impairing quality of life and increasing morbidity. The Rotterdam diagnostic criteria for PCOS are now internationally endorsed and are based on two of three features: oligo- or anovulation, hyperandrogen-

Issue Theme The International Guideline in Polycystic Ovary Syndrome: Development, Summary, and Translation for Optimal Health Impact; Guest Editors, Helena J. Teede, MBBS, FRACP, PhD and Jacqueline A. Boyle, MBBS, FRANZCOG, MPH\&TM, PhD

ism (clinical or biochemical), and polycystic ovaries. ${ }^{2}$ Insulin resistance and hyperinsulinemia play an important role in the pathophysiology and metabolic manifestations of PCOS, independent of but exacerbated by obesity. ${ }^{3}$ Early diagnosis is key in addressing symptoms, improving quality of life, and identifying fertility problems, as well as for long-term considerations including metabolic, cardiovascular, and

Copyright $\odot 2018$ by Thieme Medical Publishers, Inc., 333 Seventh Avenue, New York, NY 10001, USA. Tel: +1(212) 584-4662.
DOI https://doi.org/ 10.1055/s-0038-1668085. ISSN 1526-8004. 
psychosocial features. There is increasing evidence of the effectiveness of lifestyle interventions on nonreproductive as well as reproductive outcomes. ${ }^{4}$ Medical therapy is effective as are infertility treatments in PCOS. Here, we outline a summary of the most recent insights in diagnostic criteria, clinical features, and management of women with PCOS including a brief summary of the recommendations from the latest international evidence-based guidelines. ${ }^{5}$ The full guideline and freely available translation resources can be found at https://www.monash.edu/medicine/sphpm/mchri/ pcos and the full recommendations summary is published elsewhere. $^{1}$

\section{Diagnostic Criteria}

The Rotterdam diagnostic criteria are commonly used globally to diagnose PCOS and were the criteria endorsed by the recent international evidence-based guidelines on the diagnosis and management of PCOS. The Rotterdam diagnosis for PCOS requires two of the following features: oligo- or anovulation, clinical and/or biochemical hyperandrogenism, and polycystic ovaries. $^{2}$ In adults if oligo- or anovulation and clinical and/or biochemical hyperandrogenism are present, ultrasound may not be required. Additionally, other endocrine etiologies such as thyroid disease, nonclassic congenital adrenal hyperplasia, and hyperprolactinemia should be excluded with further evaluation guided by clinical judgement. ${ }^{5}$ The guideline refines Rotterdam criteria to highlight that in adolescents or those within 8 years of menarche, ultrasound is not a reliable discriminator for PCOS. Hence in this group, both oligo- or anovulation and clinical and/or biochemical signs of hyperandrogenism are required and ultrasound is not recommended for the purpose of diagnosis.

Ovulatory Dysfunction and Irregular Menstrual Cycles When irregular or absent menstrual cycles occur, a diagnosis of PCOS should be considered as approximately 85 to $90 \%$ of women with oligomenorrhea and 30 to $40 \%$ of women with amenorrhea have PCOS. ${ }^{6}$ Ovulatory dysfunction is assessed after 1 year post-menarche; before this time period, irregular cycles are part of the normal pubertal transition. Ovulatory dysfunction is clinically reflected by irregular menstrual cycles, which are defined as shorter than 21 days or longer than 45 days in women between 1 and 3 years post-menarche, and less than 21 or more than 35 days in women over 3 years postmenarche to perimenopause. ${ }^{5}$ Additionally, when women present with primary amenorrhea by the age of 15 or more than 3 years post-thelarche, ovulatory dysfunction is probable. It is, however, possible even with regular cycles to have ovulatory dysfunction. ${ }^{7}$ Luteal phase biochemical serum progesterone levels can be used to assess ovulation. ${ }^{5}$

\section{Hyperandrogenism}

In women with PCOS, hyperandrogenism is highly prevalent with a recent study reporting hyperandrogenism in $78 \%$ of women with PCOS and even higher prevalence in overweight women. ${ }^{8}$ When evaluating symptoms of hyperandrogenism, hirsutism should be assessed using the modified Ferriman
Gallwey score (mFG), where a level $\geq 4$ to 6 indicates hirsutism. ${ }^{5}$ Alopecia can be assessed using the Ludwig visual score. For acne, no universally accepted visual assessment is available. ${ }^{5}$ When clinical signs of hyperandrogenism are unclear or absent, measurement of calculated free testosterone, free androgen index, or calculated bioavailable testosterone should be undertaken. ${ }^{5}$ High-quality assays such as liquid chromatography-mass spectrometry/mass spectrometry and extraction/chromatography immunoassays are most accurate. Evaluation should be undertaken when women are not on hormonal contraception. ${ }^{5}$ If total or free testosterone is not elevated, consideration can be given to assessing androstenedione and dehydroepiandrosterone sulfate (DHEAS). ${ }^{5}$ In a small prospective cross-sectional study of 82 patients with PCOS, DHEAS showed higher levels in PCOS patients with PCO morphology compared with controls. ${ }^{9}$ Furthermore, a negative correlation between DHEAS levels and mean and maximum ovarian volume was reported. ${ }^{10}$ However, these findings remain unexplored in a clinical setting and there is currently no clear evidence that DHEAS is associated with ovarian morphology in PCOS.

\section{Polycystic Ovarian Morphology}

Where oligo- or anovulation and clinical and/or biochemical hyperandrogenism are present in adults, ultrasound is not strictly necessary in diagnosing PCOS; however, it will help identify the complete phenotype and may be useful for other indications in PCOS. The guideline recommends using a follicle number per ovary of more than 20 follicles (2-9 $\mathrm{mm}$ ) and/or an ovarian volume $\geq 10 \mathrm{~mL}$ using transducer frequency $\geq 8 \mathrm{MHz} .^{5}$ For older ultrasound equipment, the criterion is an ovarian volume $\geq 10 \mathrm{~mL}$. Due to the high incidence of polycystic ovaries and nonspecificity in those with a gynecological age of less than 8 years postmenarche, ultrasound is not recommended at this life stage for the purposes of diagnosis. $^{5}$

Women with polycystic ovarian morphology (PCOM) exhibit higher levels of anti-Mullerian hormone $(\mathrm{AMH})^{11}$ and higher levels of $\mathrm{AMH}$ are associated with anovulation and hyperandrogenism. ${ }^{12}$ Current research is evaluating whether serum AMH can replace ultrasound in the diagnosis of PCOM; however, the guideline does not recommend $\mathrm{AMH}$ for the detection of PCOM or the diagnosis of PCOS. Current barriers to applicability of AMH include poorly defined patient and control populations, variable quality assays, and no international standards. Additionally, there is a lack of data on clustering of AMH levels with other PCOS features and relationships to longterm outcomes and these are areas of future research. ${ }^{13}$

\section{Prevalence and Phenotypes}

The prevalence of PCOS varies depending on the diagnostic criteria, phenotypes, and populations studied. Bozdag et al reviewed a total of 55 prevalence studies. According to the diagnostic criteria of National Institutes of Health, Rotterdam, and AE-PCOS Society, the rates of PCOS prevalence were 6,10 , and $10 \%$, respectively. ${ }^{1}$ The range for prevalence on Rotterdam criteria was 8 to $13 \%$. The Rotterdam criteria have four phenotypes (-Table $\mathbf{1}$ ). The classic phenotype women 
Table 1 Polycystic ovary syndrome phenotypes

\begin{tabular}{|l|l|l|l|}
\hline Phenotype & & & \\
\hline A & $\begin{array}{l}\text { Androgen } \\
\text { excess }\end{array}$ & $\begin{array}{l}\text { Ovulatory } \\
\text { dysfunction }\end{array}$ & $\begin{array}{l}\text { Polycystic } \\
\text { ovarian } \\
\text { morphology }\end{array}$ \\
\hline B & $\begin{array}{l}\text { Androgen } \\
\text { excess }\end{array}$ & $\begin{array}{l}\text { Ovulatory } \\
\text { dysfunction }\end{array}$ & \\
\hline C & $\begin{array}{l}\text { Androgen } \\
\text { excess }\end{array}$ & $\begin{array}{l}\text { Polycystic } \\
\text { ovarian } \\
\text { morphology }\end{array}$ & \\
\hline D & $\begin{array}{l}\text { Ovulatory } \\
\text { dysfunction }\end{array}$ & $\begin{array}{l}\text { Polycystic } \\
\text { ovarian } \\
\text { morphology }\end{array}$ & \\
\hline
\end{tabular}

present with hyperandrogenism and oligomenorrhea with (A) or without (B) PCO on ultrasound. In the "ovulatory phenotype," women have hyperandrogenism and PCO (C). In the "non-hyperandrogenic phenotype," there is oligomenorrhea and PCO, without overt hyperandrogenism (D). ${ }^{14}$ Prevalence of phenotypes is variable, as this depends greatly on how the population was identified. In an Indian population, among all PCOS women, 56\% presented with phenotype A, $15 \%$ with phenotype $B, 11 \%$ with phenotype $C$, and $18 \%$ with phenotype $D$. Phenotypes A and B were seen more in obese women, with more hyperandrogenemia, insulin resistance, and worse cardiometabolic profile. Metabolic syndrome prevalence was lowest in phenotype D. ${ }^{15}$ However in other studies, these differences were not as clear. The guideline has emphasized the need for defining phenotypes in research, but the clinical relevance of this remains somewhat unclear at present.

\section{Clinical Features}

\section{Reproductive}

PCOS is reported as the cause of anovulatory infertility in 70\% of women, making it the most common cause of ovulatory dysfunction. ${ }^{16}$ In a large community-based cohort study, infertility was reported in $72 \%$ of women with PCOS compared with $16 \%$ in women without PCOS. However, encouragingly, this study also reported that women with PCOS had the same number of children as those without. ${ }^{17}$

Women with PCOS may also have an increased risk of miscarriage $^{18}$ and pregnancy complications. A recent metaanalysis found a significantly higher risk of developing gestational diabetes, pregnancy-induced hypertension, preeclampsia, preterm birth, and caesarean section. Metaregression failed to provide evidence of a significant effect between outcome and body mass index (BMI). ${ }^{19}$ Maternal complications appear to be frequent in women with hyperandrogenic PCOS compared with women with normoandrogenic PCOS. ${ }^{20}$ Women with PCOS should be informed preconception of the additional risks in pregnancy ${ }^{5}$ and health practitioners need to be aware of these increased risks. ${ }^{21}$

PCOS is also associated with an increased risk of endometrial cancer, although it remains unclear whether this risk is inde- pendent of other risk factors common in women with PCOS: obesity, diabetes, metabolic syndrome, anovulation. ${ }^{22}$ A large Danish cohort study of 12,070 women with PCOS reported a fourfold increased risk for endometrial cancer and two- to fourfold increased risks for colon, kidney, and brain tumors. They found no association between PCOS and either breast or ovarian cancer. ${ }^{23}$ Although the absolute risk of endometrial cancer is still low, health professionals should have a low threshold for excluding endometrial cancer in women with PCOS. ${ }^{5}$ When persistent thickened endometrium and/or risk factors including prolonged amenorrhea, abnormal vaginal bleeding, or excess weight are present, investigation is indicated using transvaginal ultrasound and/or endometrial biopsy. ${ }^{5}$

\section{Metabolic Syndrome}

PCOS is underpinned by insulin resistance and hyperinsulinemia. ${ }^{3}$ A recent meta-analysis of clamp assessments of insulin action in PCOS found a decrease in insulin sensitivity of $27 \%$ in women with PCOS compared with controls, independent of BMI, age, or diagnostic criteria. BMI exacerbated insulin resistance by $15 \%$ in women with PCOS and had a greater impact on insulin resistance in PCOS than in controls. ${ }^{24}$ PCOS is associated with impaired glucose tolerance (IGT) in up to $30 \%$ and type 2 diabetes in up to $10 \%$ of women with PCOS. ${ }^{25}$ When followed up over 10 years, the age-standardized prevalence of diabetes was $39.3 \%$ in women with PCOS compared with $5.0 \%$ of controls of similar age. ${ }^{26}$ When investigating differences between phenotypes, Panidis et al reported that in phenotype A, insulin resistance is more prevalent. In women with phenotype $C$, insulin resistance is not different compared with BMI-matched controls. ${ }^{27}$ Furthermore, an association between the number of ovarian follicles and insulin resistance was recently described. Ovarian follicle numbers were a significant predictor of insulin resistance in women with PCOS. ${ }^{28}$ Glycemic status should be assessed (using an oral glucose tolerance test [OGTT], fasting plasma glucose, or HbA1c) at baseline in all women with PCOS and should be repeated every 1 to 3 years depending on other individual risk factors for diabetes present. ${ }^{5}$ A 75-g OGTT is recommended for women with additional risk factors and preconception and during pregnancy.

Women with PCOS have an adverse cardiovascular risk profile including dyslipidemia, hypertension, and obstructive sleep apnea. A case-control study of 1,550 women with PCOS reported significantly lower levels of HDL and higher levels of total cholesterol, low density lipoprotein, triglycerides, and both systolic and diastolic blood pressure compared with controls, independent of BMI. ${ }^{29}$ A recent study reported that insulin and androgens may have opposing effects on lipid profiles in women with PCOS, with changes in the production of phospholipids, free fatty acids, and bioactive lipids. ${ }^{30}$ Studies have also found evidence for endothelial dysfunction in women with PCOS, matched for BMI. ${ }^{31,32}$ Furthermore, coronary artery calcification is associated with PCOS with odds ratios ranging from 2.3 to 2.4. ${ }^{33,34}$ The Coronary Artery Risk Development in Young Adults (CARDIA) study found that women with both hyperandrogenemia and oligomenorrhea (phenotype A) had an increased prevalence of coronary artery calcifications and increased carotid intima-media thickness, but women with 
isolated oligomenorrhea or hyperandrogenemia did not. ${ }^{35}$ Clinicians should also be aware that it is probable that PCOS precedes and contributes to the development of obstructive sleep apnea. However, it is possible that OSA contributes to the presentation and worsens the clinical manifestations of PCOS. Both conditions are associated with comorbidities including depression, fatigue, hypertensions, dyslipidemia, insulin resistance, and IGT. ${ }^{36}$ Women with PCOS have increased cardiovascular risk factors but evidence around long-term outcomes on cardiovascular events is limited. A recent sub-group analysis of 106 women with PCOS and 171 control women within a larger prospective cohort study of women through post-menopause (mean time (SD) since menopause 19.85 years (9.94)) reported no increased risk for stroke or ischaemic heart disease compared to the control women. (Meun et al,JCEM, 2018). However, given limited large data sets on long-term data on the morbidity and mortality for cardiovascular disease in PCOS on the background of elevated risk factors it is recommended to offer all women with PCOS a metabolic and cardiovascular assessment measuring weight, height, waist circumference, BMI, blood pressure, fasting lipid profile and monitoring of regular weight changes. ${ }^{5,37}$ If screening identifies CVD risk factors such as obesity, cigarette smoking, dyslipidemia, hypertension, IGT, and lack of physical activity, women should be considered to be at risk of CVD and screening planned as appropriate. ${ }^{5}$

\section{Psychosocial Health}

There is increasing evidence that women with PCOS are more likely to suffer from mood disorders. ${ }^{38-40}$ The underlying mechanisms are still unclear; however, factors such as obesity, insulin resistance, elevated androgens, and clinical features including distress related to hirsutism, weight gain, acne, and infertility are also drivers. ${ }^{41}$

A recent systematic review focused on moderate and severe symptoms of anxiety and depression. It reported a 4.18 increased odds of moderate or severe symptoms of depression and a 5.62 increased odds of symptoms of anxiety in women with PCOS compared with controls. The increased risk of depression in women with PCOS persisted after adjustment for BMI. Women with PCOS and symptoms of depression had higher mean values of insulin resistance, BMI, hirsutism, and infertility. ${ }^{42}$ Mood disturbances, together with other factors including self-esteem, body image, and sexual function, negatively influence quality of life in women with PCOS. ${ }^{43}$ The magnitude of psychological consequences of PCOS highlights the need for routine screening for anxiety and depressive symptoms. If the screen is positive, further assessment and/ or referral for assessment and treatment should be completed by suitably qualified health professionals. ${ }^{5}$ The PCOS qualityof-life tool (PCOSQ) may also be useful to evaluate quality of life and to highlight PCOS features that cause distress. ${ }^{5}$

\section{Treatments}

\section{Lifestyle Interventions}

Considering the implications of obesity on reproductive, metabolic, and psychosocial health, ${ }^{44}$ lifestyle interventions, including incorporating a healthy diet, increasing physical activity, and implementing behavioral strategies, are the first-line treatment for PCOS. ${ }^{45}$ Lifestyle treatment in overweight women with PCOS that leads to even small amounts of weight loss improves biochemical reproductive outcomes, surrogate markers of insulin resistance, adiposity, and adiposity distribution. ${ }^{4}$ A series of small trials show that weight loss also has a significant impact on psychosocial status and quality of life, ${ }^{46}$ hyperandrogenism, insulin resistance, lipid profiles, ovulation, menstrual cyclicity, fertility, risk factors for type 2 diabetes mellitus, and cardiovascular disease. ${ }^{45}$ Improved ovulation and live birth when lifestyle modification preceded treatment with clomiphene citrate compared with clomiphene citrate alone has also been reported. ${ }^{47}$ There is no clear evidence-based recommendations for the best dietary composition for PCOS. Significant benefits of physical exercise have been described with improvement in anthropometric measures, insulin sensitivity, lipid profile, cardiopulmonary function, inflammatory markers, and frequency of menses in women with PCOS following a structured exercise training program. ${ }^{48}$ General recommendations on exercise include 150 minutes of exercise weekly or 75 minutes of vigorous intensities weekly. For modest weight loss, prevention of weight regain, and greater health benefits, a minimum of 250 minutes weekly of moderate intensity activities or 150 minutes weekly of vigorous intensity is recommended. ${ }^{5}$ The effectiveness of lifestyle programs seems to be improved when incorporating behavioral strategies such as goal setting, self-monitoring, stimulus control, problem solving, assertiveness training, slower eating, reinforcing changes, and relapse prevention, to optimize weight management, healthy lifestyle, and emotional wellbeing. ${ }^{49}$

\section{Treatment of Nonreproductive Outcomes}

Combined oral contraceptive pills (COCPs) are recommended as first-line medical treatment for the management of hyperandrogenism and regulation of menstrual cycles in women with PCOS. ${ }^{50,51}$ The effects of COCPs are based on estrogen increasing sex hormone binding globulin (SHBG) levels which in turn decrease free testosterone and progestin, inhibiting luteinizing hormone ( $\mathrm{LH})$ secretion resulting in a decline in androgen production and suppression of $5-\alpha$ reductase activity which reduces conversion of T to DHT. ${ }^{52}$ Prolonged use of COCPs is effective in decreasing $\mathrm{mFG}$ score, total testosterone, free testosterone, androstenedione, and DHEAS and increasing SHBG. ${ }^{52}$ Rates of depression and anxiety have not shown a significant change after treatment with COCPs in PCO; however, studies in general populations have noted an impact of the COCP on libido and mood. ${ }^{53}$ Previous studies have raised concerns of COCPs affecting insulin resistance and triglycerides; however, a recent overview of systematic reviews found no evidence of increased cardiovascular events in prolonged use of COCPs. ${ }^{54}$ There is limited information on which COCP preparation type or duration of therapy is optimal for women with PCOS, with all agents reducing SHBG and improving clinical outcomes. Therefore, to minimize complications and based on general population data, recommendations are to use the lowest effective estrogen dose and natural estrogen preparations, balancing efficacy, metabolic risk profile, side effects, costs, 
and availability. ${ }^{5}$ In combination with COCPs, metformin could be used to avoid weight gain ${ }^{55}$ and further suppress hyperandrogenism. ${ }^{56}$

Because of the metabolic features of PCOS such as insulin resistance and hyperinsulinemia, insulin-sensitizing agents, especially metformin, have been used as a treatment option for PCOS for several decades. ${ }^{57}$ Metformin reduces insulin levels leading to a reduction of ovarian and adrenal secretions of androgens, pituitary secretion of LH, and increase of SHBG levels. ${ }^{58}$ This mechanism may lead to the favorable effect metformin has on BMI, ${ }^{59-61}$ menstrual frequency, ${ }^{59,62}$ systolic blood pressure, ${ }^{61,62}$ fasting glucose levels, ${ }^{62}$ fasting insulin levels, ${ }^{62}$ testosterone, ${ }^{59,61,62}$ triglyceride levels, ${ }^{61}$ and $\mathrm{LH}$ levels. ${ }^{59}$ Results, however, do show high levels of heterogeneity. An effect has not been reported on $\mathrm{mFG}$ scores, ${ }^{61}$ cholesterol levels, ${ }^{61,62}$ or free androgen index. ${ }^{61}$ A systematic review and meta-analysis demonstrated that the combination of lifestyle modification and metformin use is associated with lower BMI, subcutaneous adipose tissue, and improved menstruation compared with the combination of lifestyle and placebo. ${ }^{60}$ Hence, metformin may be used as an adjuvant to lifestyle modification but not as a substitute for it. When combined with lifestyle intervention, metformin may have an additive effect in improving cardio metabolic risk, especially in high metabolic risk groups. Hence, metformin use is recommended in PCOS for weight and metabolic effects. Commencement at a lower dose is recommended with subsequent titration $^{5}$ to minimize mild and self-limiting side effects.

The role of antiandrogens in the treatment of hirsutism in PCOS is controversial; however, there is some low-quality evidence that flutamide and spironolactone reduce hirsutism. ${ }^{63}$ A recent study showed a higher reduction in hirsutism when COCPs were used in combination with the antiandrogen bicalutamide compared with COCP alone. ${ }^{64}$ In combination with COCPs, antiandrogens should only be considered to treat hirsutism after a minimum of 6 months of COCPs after cosmetic therapy has failed. ${ }^{5}$ However, most of these adjuvant medications are used off label and are not registered for the treatment of hyperandrogenism.

Bariatric surgery can improve menstrual irregularity, hirsutism, and infertility as reported in a recent systematic review and meta-analysis. The incidence of PCOS decreased by nearly $40 \%$ at study endpoint, with nearly $50 \%$ improvement of menstrual irregularity and 30\% improvement in hirsutism. ${ }^{65}$ However, registry studies demonstrate concerns around pregnancy outcomes. For this reason, the guidelines recommend that bariatric surgery can be considered in PCOS after lifestyle therapy fails; however, it should not be recommended for the purpose of improving fertility outcomes pending further research.

\section{Treatment of Reproductive Outcomes}

Interventions in fertility management should be preceded by the optimization of lifestyle and psychological health to improve reproductive and obstetric outcomes. ${ }^{5}$ Pharmacological options include ovulation induction with letrozole, clomiphene citrate (CC) metformin, and gonadotrophins. Letrozole is considered first-line treatment for women with
PCOS and anovulatory infertility. Letrozole followed by timed intercourse improves live birth rate and clinical pregnancy rates compared with $\mathrm{CC}$ with no difference in rates of ovarian hyperstimulation syndrome (OHSS) and reduced multiple pregnancy. ${ }^{66}$ Regulations, however, vary internationally on use of letrozole for treatment of infertility and treatment is usually off-label requiring explanation and consent.

CC is recommended for infertility treatment in PCOS both alone and with metformin. Pregnancy and live birth rates are increased, but is associated with increased multiple pregnancy rates $(5-8 \%)$ and OHSS $(<1 \%) .{ }^{67}$ There is also evidence of a benefit from metformin on clinical pregnancy rates and ovulation rates compared with placebo and when combined with CC compared with CC alone. ${ }^{62}$ Literature also suggests that metformin is effective in nonobese women. ${ }^{62} \mathrm{CC}$ could therefore be combined with metformin, rather than persisting with CC alone, especially in women with PCOS who are CC resistant.

Stimulating follicle development and growth with exogenous gonadotrophins for ovulation induction in women with CC-resistant PCOS is well established, ${ }^{68}$ and could be used as second-line pharmacological treatment when firstline therapy has failed. Addition of metformin may increase live birth rate among women undergoing ovulation induction with gonadotrophins. ${ }^{69}$

For anovulatory infertility in women who are clomiphene resistant, laparoscopic ovarian drilling is a successful secondline treatment for ovulation induction. ${ }^{70}$ It offers several advantages including avoiding OHSS and multiple pregnancies, negating the need for complex monitoring, the favorable effect on physiological ovulatory cycles, and the possible long-term reproductive and endocrine benefits. ${ }^{71}$

In vitro fertilization (IVF) is indicated for women with anovulatory PCOS after failure to respond to ovulation induction or if there are other indications such as tubal damage or male subfertility. ${ }^{72}$ A gonadotrophin-releasing hormone antagonist protocol is favored over an agonist protocol because of the reduced OHSS risk. ${ }^{5}$ When an agonist protocol is used, metformin could improve clinical pregnancy rate and reduce OHSS risk. ${ }^{5}$

\section{Summary}

Polycystic ovary syndrome is a complex reproductive, metabolic, and psychological condition that affects women's health and quality of life across the life-course. Physicians should be aware of the clinical features and risks for women with PCOS and screen and manage accordingly. Clinicians should focus on lifestyle adjustments as the first-line management to improve reproductive, metabolic, cardiovascular, and psychosocial outcomes focusing on weight management and physical exercise. In addition, pharmacological therapy in the form of COCPs and metformin may be useful. For the management of anovulatory infertility with no other factors, lifestyle intervention is recommended as the first-line management. If this is unsuccessful, ovulation induction using letrozole is the first-line medical management, and after this clomiphene citrate and metformin may have an additive effect. Gonadotrophins and laparoscopic surgery are second line and IVF therapy is the third-line therapy 
where other treatments have failed. The latest international evidence-based guidelines detail these treatments along with providing translation resources for health professional or women with PCOS. Together these are designed to provide a valuable resource aiding clinicians in optimal assessment and management of PCOS.

\section{Funding}

H.J.T. and J.A.B. are NHMRC-funded research fellows.

\section{Conflicts of Interest}

The authors have no conflicts of interest to disclose.

\section{References}

1 Bozdag G, Mumusoglu S, Zengin D, Karabulut E, Yildiz BO. The prevalence and phenotypic features of polycystic ovary syndrome: a systematic review and meta-analysis. Hum Reprod 2016;31(12):2841-2855

2 Rotterdam ESHRE/ASRM-Sponsored PCOS Consensus Workshop Group. Revised 2003 consensus on diagnostic criteria and longterm health risks related to polycystic ovary syndrome. Fertil Steril 2004;81(01):19-25

3 Dumesic DA, Oberfield SE, Stener-Victorin E, Marshall JC, Laven JS, Legro RS. Scientific statement on the diagnostic criteria, epidemiology, pathophysiology, and molecular genetics of polycystic ovary syndrome. Endocr Rev 2015;36(05):487-525

4 Moran LJ, Hutchison SK, Norman RJ, Teede HJ. Lifestyle changes in women with polycystic ovary syndrome. Cochrane Database Syst Rev 2011;16(2)

5 Teede HJ, Misso ML, Costello MF, et al. Norman RJ on behalf of the International PCOS Network. Recommendations from the international evidence-based guideline for the assessment and management of polycystic ovary syndrome. Fertil Steril 2018; In press

6 Hart R. Definitions, prevalence and symptoms of polycystic ovaries and polycystic ovary syndrome. In: Allahbadia G, Agrawal R, eds. Polycystic Ovary Syndrome. 2nd ed. Kent, UK: Anshan, Ltd; 2007:15-26

7 Balen AH, Conway GS, Kaltsas G, et al. Polycystic ovary syndrome: the spectrum of the disorder in 1741 patients. Hum Reprod 1995; 10(08):2107-2111

8 Alexiou E, Hatziagelaki E, Pergialiotis V, et al. Hyperandrogenemia in women with polycystic ovary syndrome: prevalence, characteristics and association with body mass index. Horm Mol Biol Clin Investig 2017;29(03):105-111

9 Tena G, Moran C, Romero R, Moran S. Ovarian morphology and endocrine function in polycystic ovary syndrome. Arch Gynecol Obstet 2011;284(06):1443-1448

10 Christodoulaki C, Trakakis E, Pergialiotis V, et al. Dehydroepiandrosterone-sulfate, insulin resistance and ovarian volume estimation in patients with polycystic ovarian syndrome. J Family Reprod Health 2017;11(01):24-29

11 Piouka A, Farmakiotis D, Katsikis I, Macut D, Gerou S, Panidis D. Anti-Mullerian hormone levels reflect severity of PCOS but are negatively influenced by obesity: relationship with increased luteinizing hormone levels. Am J Physiol Endocrinol Metab 2009;296(02):E238-E243

12 Alebić MS, Stojanović N, Duhamel A, Dewailly D. The phenotypic diversity in per-follicle anti-Müllerian hormone production in polycystic ovary syndrome. Hum Reprod 2015;30(08): 1927-1933

13 Pankhurst MW, Shorakae S, Rodgers RJ, Teede HJ, Moran LJ. Efficacy of predictive models for polycystic ovary syndrome using serum levels of two antimüllerian hormone isoforms (proAMH and $\mathrm{AMH}_{\mathrm{N}, \mathrm{C}}$ ). Fertil Steril 2017;108(05):851-857.e2

14 Spritzer PM. Polycystic ovary syndrome: reviewing diagnosis and management of metabolic disturbances. Arq Bras Endocrinol Metabol 2014;58(02):182-187

15 Tripathy P, Sahu A, Sahu M, Nagy A. Metabolic risk assessment of Indian women with polycystic ovarian syndrome in relation to four Rotterdam criteria based phenotypes. Eur J Obstet Gynecol Reprod Biol 2018;224:60-65

16 Brassard M, AinMelk Y, Baillargeon JP. Basic infertility including polycystic ovary syndrome. Med Clin North Am 2008;92(05): 1163-1192, xi

17 Joham AE, Teede HJ, Ranasinha S, Zoungas S, Boyle J. Prevalence of infertility and use of fertility treatment in women with polycystic ovary syndrome: data from a large community-based cohort study. J Womens Health (Larchmt) 2015;24(04):299-307

18 Rees DA, Jenkins-Jones S, Morgan CL. Contemporary reproductive outcomes for patients with polycystic ovary syndrome: a retrospective observational study. J Clin Endocrinol Metab 2016;101 (04):1664-1672

19 Qin JZ, Pang LH, Li MJ, Fan XJ, Huang RD, Chen HY. Obstetric complications in women with polycystic ovary syndrome: a systematic review and meta-analysis. Reprod Biol Endocrinol 2013;11(11):56

20 de Wilde MA, Lamain-de Ruiter M, Veltman-Verhulst SM, et al. Increased rates of complications in singleton pregnancies of women previously diagnosed with polycystic ovary syndrome predominantly in the hyperandrogenic phenotype. Fertil Steril 2017;108(02):333-340

21 Boomsma CM, Eijkemans MJ, Hughes EG, Visser GH, Fauser BC, Macklon NS. A meta-analysis of pregnancy outcomes in women with polycystic ovary syndrome. Hum Reprod Update 2006;12 (06):673-683

22 Barry JA, Azizia MM, Hardiman PJ. Risk of endometrial, ovarian and breast cancer in women with polycystic ovary syndrome: a systematic review and meta-analysis. Hum Reprod Update 2014; 20(05):748-758

23 Gottschau M, Kjaer SK, Jensen A, Munk C, Mellemkjaer L. Risk of cancer among women with polycystic ovary syndrome: a Danish cohort study. Gynecol Oncol 2015;136(01):99-103

24 Cassar S, Misso ML, Hopkins WG, Shaw CS, Teede HJ, Stepto NK. Insulin resistance in polycystic ovary syndrome: a systematic review and meta-analysis of euglycaemic-hyperinsulinaemic clamp studies. Hum Reprod 2016;31(11):2619-2631

25 Legro RS, Kunselman AR, Dodson WC, Dunaif A. Prevalence and predictors of risk for type 2 diabetes mellitus and impaired glucose tolerance in polycystic ovary syndrome: a prospective, controlled study in 254 affected women. J Clin Endocrinol Metab 1999;84(01):165-169

26 Gambineri A, Patton L, Altieri P, et al. Polycystic ovary syndrome is a risk factor for type 2 diabetes: results from a long-term prospective study. Diabetes 2012;61(09):2369-2374

27 Panidis D, Tziomalos K, Misichronis G, et al. Insulin resistance and endocrine characteristics of the different phenotypes of polycystic ovary syndrome: a prospective study. Hum Reprod 2012;27 (02):541-549

28 Hong SH, Sung YA, Hong YS, Jeong K, Chung H, Lee H. Polycystic ovary morphology is associated with insulin resistance in women with polycystic ovary syndrome. Clin Endocrinol (Oxf) 2017;87 (04):375-380

29 Pinola P, Puukka K, Piltonen TT, et al. Normo- and hyperandrogenic women with polycystic ovary syndrome exhibit an adverse metabolic profile through life. Fertil Steril 2017;107 (03):788-795.e2

30 Li S, Chu Q Ma J, et al. Discovery of novel lipid profiles in PCOS: do insulin and androgen oppositely regulate bioactive lipid production? J Clin Endocrinol Metab 2017;102(03):810-821 
31 Kravariti M, Naka KK, Kalantaridou SN, et al. Predictors of endothelial dysfunction in young women with polycystic ovary syndrome. J Clin Endocrinol Metab 2005;90(09):5088-5095

32 Paradisi G, Steinberg HO, Hempfling A, et al. Polycystic ovary syndrome is associated with endothelial dysfunction. Circulation 2001;103(10):1410-1415

33 Christian RC, Dumesic DA, Behrenbeck T, Oberg AL, Sheedy PF II, Fitzpatrick LA. Prevalence and predictors of coronary artery calcification in women with polycystic ovary syndrome. J Clin Endocrinol Metab 2003;88(06):2562-2568

34 Talbott EO, Zborowski JV, Rager JR, Boudreaux MY, Edmundowicz DA, Guzick DS. Evidence for an association between metabolic cardiovascular syndrome and coronary and aortic calcification among women with polycystic ovary syndrome. J Clin Endocrinol Metab 2004;89(11):5454-5461

35 Calderon-Margalit R, Siscovick D, Merkin SS, et al. Prospective association of polycystic ovary syndrome with coronary artery calcification and carotid-intima-media thickness: the Coronary Artery Risk Development in Young Adults Women's study. Arterioscler Thromb Vasc Biol 2014;34(12):2688-2694

36 Kahal H, Kyrou I, Tahrani AA, Randeva HS. Obstructive sleep apnoea and polycystic ovary syndrome: a comprehensive review of clinical interactions and underlying pathophysiology. Clin Endocrinol (Oxf) 2017;87(04):313-319

37 Meun C, Franco OH, Dhana K, et al. High androgens in postmenopausal women and the risk for atherosclerosis and cardiovascular disease: the Rotterdam study. J Clin Endocrinol Metab 2018; 103(04):1622-1630. doi: 10.1210/jc.2017-02421

38 Barry JA, Kuczmierczyk AR, Hardiman PJ. Anxiety and depression in polycystic ovary syndrome: a systematic review and metaanalysis. Hum Reprod 2011;26(09):2442-2451

39 Dokras A, Clifton S, Futterweit W, Wild R. Increased risk for abnormal depression scores in women with polycystic ovary syndrome: a systematic review and meta-analysis. Obstet Gynecol 2011;117(01):145-152

40 Veltman-Verhulst SM, Boivin J, Eijkemans MJ, Fauser BJ. Emotional distress is a common risk in women with polycystic ovary syndrome: a systematic review and meta-analysis of 28 studies. Hum Reprod Update 2012;18(06):638-651

41 Farrell K, Antoni MH. Insulin resistance, obesity, inflammation, and depression in polycystic ovary syndrome: biobehavioral mechanisms and interventions. Fertil Steril 2010;94(05):1565-1574

42 Cooney LG, Lee I, Sammel MD, Dokras A. High prevalence of moderate and severe depressive and anxiety symptoms in polycystic ovary syndrome: a systematic review and meta-analysis. Hum Reprod 2017;32(05):1075-1091

43 Bazarganipour F, Ziaei S, Montazeri A, Foroozanfard F, Kazemnejad A, Faghihzadeh S. Health-related quality of life in patients with polycystic ovary syndrome (PCOS): a model-based study of predictive factors. J Sex Med 2014;11(04):1023-1032

44 Lim SS, Norman RJ, Davies MJ, Moran LJ. The effect of obesity on polycystic ovary syndrome: a systematic review and meta-analysis. Obes Rev 2013;14(02):95-109

45 Teede H, Michelmore J, McCallister V, Norman R. Evidence- based guidelines in PCOS. 2011. Available at: www.nhmrc.gov.au/ guidelines/publications/ext2. Accessed July 17, 2018

46 Dokras A, Sarwer DB, Allison KC, et al. Weight loss and lowering androgens predict improvements in health-related quality of life in women with PCOS. J Clin Endocrinol Metab 2016;101(08): 2966-2974

47 Legro RS, Dodson WC, Kunselman AR, et al. Benefit of delayed fertility therapy with preconception weight loss over immediate therapy in obese women with PCOS. J Clin Endocrinol Metab 2016;101(07):2658-2666

48 Orio F, Muscogiuri G, Giallauria F, et al. Oral contraceptives versus physical exercise on cardiovascular and metabolic risk factors in women with polycystic ovary syndrome: a randomized controlled trial. Clin Endocrinol (Oxf) 2016;85(05):764-771
49 Brennan L, Teede H, Skouteris H, Linardon J, Hill B, Moran L. Lifestyle and behavioral management of polycystic ovary syndrome. J Womens Health (Larchmt) 2017;26(08):836-848

50 Helvaci N, Yildiz BO. Oral contraceptives in polycystic ovary syndrome. Minerva Endocrinol 2014;39(03):175-187

51 Escobar-Morreale HF, Carmina E, Dewailly D, et al. Epidemiology, diagnosis and management of hirsutism: a consensus statement by the Androgen Excess and Polycystic Ovary Syndrome Society. Hum Reprod Update 2012;18(02):146-170

52 Amiri M, Kabir A, Nahidi F, Shekofteh M, Ramezani Tehrani F. Effects of combined oral contraceptives on the clinical and biochemical parameters of hyperandrogenism in patients with polycystic ovary syndrome: a systematic review and meta-analysis. Eur J Contracept Reprod Health Care 2018;23(01):64-77

53 Cinar N, Harmanci A, Demir B, Yildiz BO. Effect of an oral contraceptive on emotional distress, anxiety and depression of women with polycystic ovary syndrome: a prospective study. Hum Reprod 2012;27(06):1840-1845

54 Tay CT, Joham AE, Hiam DS, et al. Pharmacological and surgical treatment of non-reproductive outcomes in polycystic ovary syndrome: an overview of systematic reviews. Clin Endocrinol (Oxf) 2018. Doi: 10.1111/cen.13753. [Epub ahead of print]

55 Glintborg D, Altinok ML, Mumm H, Hermann AP, Ravn P, Andersen M. Body composition is improved during 12 months' treatment with metformin alone or combined with oral contraceptives compared with treatment with oral contraceptives in polycystic ovary syndrome. J Clin Endocrinol Metab 2014;99(07):2584-2591

56 Elter K, Imir G, Durmusoglu F. Clinical, endocrine and metabolic effects of metformin added to ethinyl estradiol-cyproterone acetate in non-obese women with polycystic ovarian syndrome: a randomized controlled study. Hum Reprod 2002;17(07): 1729-1737

57 Velazquez EM, Mendoza S, Hamer T, Sosa F, Glueck CJ. Metformin therapy in polycystic ovary syndrome reduces hyperinsulinemia, insulin resistance, hyperandrogenemia, and systolic blood pressure, while facilitating normal menses and pregnancy. Metabolism 1994;43(05):647-654

58 Diamanti-Kandarakis E, Christakou CD, Kandaraki E, Economou FN. Metformin: an old medication of new fashion: evolving new molecular mechanisms and clinical implications in polycystic ovary syndrome. Eur J Endocrinol 2010;162(02):193-212

59 Yang PK, Hsu CY, Chen MJ, et al. The efficacy of 24-month metformin for improving menses, hormones, and metabolic profiles in polycystic ovary syndrome. J Clin Endocrinol Metab 2018;103(03):890-899

60 Naderpoor N, Shorakae S, de Courten B, Misso ML, Moran LJ, Teede HJ. Metformin and lifestyle modification in polycystic ovary syndrome: systematic review and meta-analysis. Hum Reprod Update 2015;21(05):560-574

61 Patel R, Shah G. Effect of metformin on clinical, metabolic and endocrine outcomes in women with polycystic ovary syndrome: a meta-analysis of randomized controlled trials. Curr Med Res Opin 2017;33(09):1545-1557

62 Morley LC, Tang T, Yasmin E, Norman RJ, Balen AH. Insulinsensitising drugs (metformin, rosiglitazone, pioglitazone, Dchiro-inositol) for women with polycystic ovary syndrome, oligo amenorrhoea and subfertility. Cochrane Database Syst Rev 2017; 11(11):CD003053

63 van Zuuren EJ, Fedorowicz Z, Carter B, Pandis N. Interventions for hirsutism (excluding laser and photoepilation therapy alone). Cochrane Database Syst Rev 2015;28(04):CD010334

64 Moretti C, Guccione L, Di Giacinto P, et al. Combined oral contraception and bicalutamide in polycystic ovary syndrome and severe hirsutism: a double-blind randomized controlled trial. J Clin Endocrinol Metab 2018;103(03):824-838

65 Skubleny D, Switzer NJ, Gill RS, et al. The impact of bariatric surgery on polycystic ovary syndrome: a systematic review and meta-analysis. Obes Surg 2016;26(01):169-176 
66 Franik S, Eltrop SM, Kremer JAM, Kiesel L, Farquhar C. Aromatase inhibitors (letrozole) for subfertile women with polycystic ovary syndrome. Cochrane Database Syst Rev 2018;5:CD010287

67 Kafy S, Tulandi T. New advances in ovulation induction. Current Opinion in Obstetrics \& Gynecology 2007;19:248-252

68 Weiss NS, Nahuis M, Bayram N, Mol BW, Van der Veen F, van Wely M. Gonadotrophins for ovulation induction in women with polycystic ovarian syndrome. Cochrane Database Syst Rev 2015;9(09): CD010290

69 Bordewijk EM, Nahuis M, Costello MF, et al. Metformin during ovulation induction with gonadotrophins followed by timed intercourse or intrauterine insemination for subfertility associated with polycystic ovary syndrome. Cochrane Database Syst Rev 2017;1(01):CD009090
70 Farquhar C, Brown J, Marjoribanks J. Laparoscopic drilling by diathermy or laser for ovulation induction in anovulatory polycystic ovary syndrome. Cochrane Database Syst Rev 2012;(06): CD001122

71 Nahuis MJ, Oude Lohuis E, Kose N, et al. Long-term follow-up of laparoscopic electrocautery of the ovaries versus ovulation induction with recombinant FSH in clomiphene citrate-resistant women with polycystic ovary syndrome: an economic evaluation. Hum Reprod 2012;27(12):3577-3582

72 Balen AH, Morley LC, Misso M, et al. The management of anovulatory infertility in women with polycystic ovary syndrome: an analysis of the evidence to support the development of global WHO guidance. Hum Reprod Update 2016;22(06): 687-708 